\title{
Dosimetric comparison between coplanar and non coplanar field radiotherapy for ethmoid sinus cancer
} Antoine Serre ${ }^{1}$, Katia Idri², Pascal Fenoglietto ${ }^{2}$, Norbert Ailleres², Lore Santoro ${ }^{2}$, Claire Lemanski ${ }^{1}$, Renaud Garrel ${ }^{3}$, Marc Makeieff ${ }^{3}$, Ali Allaw ${ }^{1}$, Jean-Bernard Dubois ${ }^{1}$ and David Azria*1

Address: ${ }^{1}$ Department of Radiation Oncology, Val d'Aurelle Cancer Institute, Montpellier, France, ${ }^{2}$ Radiophysics Unit, Val d'Aurelle Cancer Institute, Montpellier, France and ${ }^{3}$ Department of Head and Neck Surgery, University Hospital Gui De Chauliac, Montpellier, France

Email: Antoine Serre - Antoine.Serre@valdorel.fnclcc.fr; Katia Idri - Katia.Idri@valdorel.fnclcc.fr;

Pascal Fenoglietto - Pascal.Fenoglietto@valdorel.fnclcc.fr; Norbert Ailleres - Norbert.Ailleres@valdorel.fnclcc.fr;

Lore Santoro - Lore.Santoro@valdorel.fnclcc.fr; Claire Lemanski - Claire.Lemanski@valdorel.fnclcc.fr; Renaud Garrel - r-garrel@chumontpellier.fr; Marc Makeieff - m.makeieff@libertysurf.fr; Ali Allaw - aliallaw@hotmail.com; Jean-Bernard Dubois - jeanbernard.dubois@valdorel.fnclcc.fr; David Azria* - azria@valdorel.fnclcc.fr

* Corresponding author

Published: 18 September 2007

Radiation Oncology 2007, 2:35 doi:10.1186/1748-717X-2-35
Received: 27 July 2007

Accepted: 18 September 2007

This article is available from: http://www.ro-journal.com/content/2/1/35

(c) 2007 Serre et al; licensee BioMed Central Ltd.

This is an Open Access article distributed under the terms of the Creative Commons Attribution License (http://creativecommons.org/licenses/by/2.0), which permits unrestricted use, distribution, and reproduction in any medium, provided the original work is properly cited.

\begin{abstract}
Background: To compare non coplanar field (NCF) with coplanar field (CF) -intensity-modulated radiotherapy (IMRT) planning for ethmoid cancer.

Methods: Seven patients treated with NCF IMRT for ethmoid cancer were studied. A CF IMRT optimization was prepared with the same constraints as for the NCF treatment. The maximum point doses ( $D$ max) obtained for the different optic pathway structures (OPS) should differ no more than $3 \%$ from those achieved with the NCF IMRT plan. The distribution of the dose in the target volume and in the critical structures was compared between the two techniques, as well as the Conformity $(\mathrm{Cl})$ and the Homogeneity Indexes $(\mathrm{HI})$ in the target volume.
\end{abstract}

Results: We noted no difference between the two techniques in the OPS for the DI, D2, and $\mathrm{D} 5 \%$, in the inner ear and controlateral lens for the average Dmax, in the temporo-mandibular joints for the average mean dose, in the cord and brainstem for the average DI\%. The dose-volume histograms were slightly better with the NCF treatment plan for the planning target volume (PTV) with a marginally better $\mathrm{HI}$ but no impact on $\mathrm{Cl}$. We found a great improvement in the PTV coverage with the CF treatment plan for two patients with T4 tumors.

Conclusion: IMRT is one of the treatment options for ethmoid cancer. The PTV coverage is optimal without compromising the protection of the OPS. The impact of non coplanar versus coplanar set up is very slight.

\section{Background}

Ethmoid sinus cancers are rare malignant tumors of the paranasal sinuses. They are often diagnosed at a late stage and are often, at that point, locally advanced. Despite the lack of randomised studies [1-3] we have a multidisciplinary approach with initial surgery and adjuvant radiother- 
apy. Planning the radiation treatment is a challenge for the radiophysician due to the proximity of critical and radiosensitive structures. The outcome is suboptimal with locoregional failure and treatment morbidity [4-9]. The implementation of intensity modulated radiotherapy (IMRT) for this pathology offers a better bilateral sparing of the optic pathways and probably increases the therapeutic ratio. Nevertheless, the coverage of the target volume depends on the dose delivered to optic structure [10]. In daily therapeutic practice, the physician has to make a decision based on the probabilities for locoregional control and the risks of loosing binocular vision. The possible contribution of non coplanar field (NCF) as opposed to coplanar field (CF) IMRT is not well known. In our hospital, 7 patients were treated for ethmoid sinus carcinoma by IMRT with a non coplanar technique. The dose delivered to the optic structures depended on initial staging of the pathology and was at the discretion of the physician. For all these patients, we came up with a coplanar treatment plan, with the same maximum doses to optic structures as those obtained with NCF. The aim of this paper was to compare the dose distribution in the target volumes and in other various critical structures in coplanar and non coplanar field IMRT.

\section{Methods \\ Patients}

Between July 2004 and April 2005, 7 consecutive patients (3 males and 4 females) with node-negative ethmoid sinus tumors (based on the CT scan) were treated in the radiotherapy department of the CRLC Val D'Aurelle Paul Lamarque. The median age was 51.7 years old (range 24 to 72 years). The initial staging was performed clinically with a cervico-facial CT scan, a sinus MRI, a chest Xray and an abdominal US scan. We detected three adenocarcinomas, three esthesioneuroblastomas and one undifferentiated neuroendocrine carcinoma. Four patients were treated with surgery and post operative radiotherapy, two patients with concomitant radio-chemotherapy and one with sequential radio-chemotherapy. The studied population consisted of three T2, two T3 and two T4; one with anterior orbital soft tissue invasion, the other with extensions in the cavernous sinus.

\section{Patient data acquisition}

Patients were immobilized in supine position with a customized cushion (Moldcare, Bebig ${ }^{\circledR}$ ) and a 5-point thermoplastic face mask. A CT scan (Picker PQ2000) with iodine injection was performed from the vertex to the sternum with $3 \mathrm{~mm}$ slice thickness spaced every $3 \mathrm{~mm}$. Images were transferred to the virtual simulation system (Acqsim Philips) and the isocenter, located in the clinical target volume (CTV1), was defined directly after the CT scan acquisition. This referent point was marked on the patient face mask using mobile lasers.

\section{Contouring of target volumes}

For non-operated patients, the gross tumor volume (GTV) was delineated after MRI-CT fusion scan. The CTV1 was defined with a 3D empiric margin of $4 \mathrm{~mm}$ around the GTV. For operated patients, the CTV1 was contoured after the same fusion scan with the help of the surgical report and the anatomo-pathologist's results. The CTV2 included all the CTV1, the ethmoid, the ipsilateral maxillary sinus, the nasal cavity, the sphenoid sinus and the caudal part of the frontal sinus. In the case of sphenoid invasion, the cavernous sinus was included and for orbit extension the whole orbit was delineated. The planning target volume (PTV) was determined by a $4 \mathrm{~mm} 3 \mathrm{D}$ margin around the CTV.

\section{Contouring of organs at risk}

For all patients, the following structures were delineated: spinal cord, extended spinal cord (ext cord) made up of a $7 \mathrm{~mm}$ 3D margin, brainstem, frontal and parietal lobes, pituitary gland, optic chiasma, optic nerves, retinae, lenses increased by a $2 \mathrm{~mm}$ 3D safety margin, parotids, temporomandibular joints, lachrymal glands.

\section{Treatment protocol with non coplanar fields}

The 7 patients were treated with 5 non coplanar fields: two fields with $95^{\circ}$ and $265^{\circ}$ gantry angulations without table rotation and three non coplanar fields with $35^{\circ}$, $320^{\circ}$ and $345^{\circ}$ gantry angulations with $90^{\circ}$ table rotation.

Optimization was performed on the inverse planning system Eclipse Helios (Varian Medical Systems) with $6 \mathrm{MV}$ photon beams. The dose constraints used for the different volumes are summarized in Table 1.

The dose delivered to the optic pathways depended on the proximity of the CTV1. Therefore, to validate the treatment plan, the physician had to compromise between target volume coverage and the dose to the optic pathways.

\section{Comparative study using coplanar fields}

The comparative study consisted of performing a treatment plan for each patient using coplanar fields with a maximum delivered dose (Dmax) to the chiasma and optic nerves within 3\% of the Dmax delivered with the non coplanar treatment plan. Optimization was performed with the same inverse planning system using the set of constraints described in Table 1 . Five $6 \mathrm{MV}$ photon coplanar fields were applied to these patients with $0^{\circ}$, $70^{\circ}, 140^{\circ}, 210^{\circ}$ and $290^{\circ}$ gantry angulations, the latter being approved by the physician in charge of the patient.

The dose-volume histograms (DVH) were calculated for all the delineated volumes in the two different treatment plans. Conformity and homogeneity indices were calcu- 
Table I: Dose-volume constraint set used for inverse planning optimization

\begin{tabular}{ll}
\hline Volume & Constraint \\
\hline PTV & V $95 \%>95 \%$ prescribed dose \\
CTV & V $99 \%>95 \%$ prescribed dose \\
Spinal cord & D max $<40 \mathrm{~Gy}$ \\
Ext cord & D max $<45 \mathrm{~Gy}$ \\
Brainstem & $\mathrm{D} \max <55 \mathrm{~Gy}$ \\
Frontal lob & $\mathrm{D} \max <60 \mathrm{~Gy}$ \\
Parietal lob & $\mathrm{D} \max <60 \mathrm{~Gy}$ \\
Hypophyse & $\mathrm{D} \max <55 \mathrm{~Gy}$ \\
Temporomandibular joints & $\mathrm{D} \max <60 \mathrm{~Gy}$ \\
Parotid & $\mathrm{D} \operatorname{mean}<26 \mathrm{~Gy}$ \\
& V $50 \%<30 \mathrm{~Gy}$ \\
Lens & $\mathrm{D} \max <12 \mathrm{~Gy}$ \\
Optic nerve & $\mathrm{D} \max <55 \mathrm{~Gy}$ \\
Chiasma & $\mathrm{D} \max <55 \mathrm{~Gy}$ \\
Retina & $\mathrm{D} \max <55 \mathrm{~Gy}$ \\
Lachrymal glands & No constraint \\
\hline
\end{tabular}

lated for the PTV1 [11]. Homogeneity index (HI) is defined by the difference between D1 and D99\% divided by the prescribed dose. Conformity index (CI) is defined as follows:

$$
\mathrm{CI}=\left(\mathrm{TV} / \mathrm{V}_{\mathrm{PTV}}\right) \times\left(\mathrm{TV} / \mathrm{V}_{95 \%}\right)
$$

TV: Treated Volume is the volume of PTV1 receiving the prescribed dose (95\%)

$\mathrm{V}_{\mathrm{PTV}}$ is the volume of PTV

$\mathrm{V}_{95 \%}$ is the volume enclosed in the isodose $95 \%$

\section{Results}

\section{Dose distribution in the optic pathways}

The maximum dose in the optic chiasma, ipsilateral and controlateral optic nerves are represented in the Figure $1 \mathrm{a}, \mathrm{b}, \mathrm{c}$. No difference was noted between NCF and CF techniques.

Likewise, the doses delivered in 1, 2 and 5\% of these volumes (D1, D2 and D5\%) were similar for the two techniques (Figure 2a,b,c). The differences did not exceed $2 \mathrm{~Gy}$ except for patient $\mathrm{n}^{\circ} 2$, where the dose in the controlateral optic nerve was more than 3 Gy lower with the CF technique.

\section{Dose distribution in organs at risk}

In Figure 3, we show the most characteristic tolerance doses for each organ at risk (OAR). Also represented are the mean values for each organ with their standard deviation. No difference was noted between CF and NCF for the ipsilateral and controlateral lenses, brainstem, temporomandibular joints, inner ears or parotids. Moreover, we did not find a subgroup of patients who benefited from either of the techniques.

\section{Dose distribution in the target volume}

The mean dose volume histogram for the PTV1 is shown in Figure 4. We can observe a slight difference in favour of the NCF compared with the CF. The HI confirm this tendency with a mean value of 0.14 for the NCF and 0.16 for the CF; HIs are represented for each patient in Figure 5. The conformity index for the two techniques was similar as shown in Figure 6. In the subgroup of patients with skull base involvement (T4), a significant benefit was noticed with the CF compared to the NCF (Fig. 7). Ninety percent and $95 \%$ of the volume received respectively $96 \%$ and $94.5 \%$ of the prescribed dose with the CF technique and $94.5 \%$ and $93.5 \%$ with the NCF technique.

In the other subgroups, we observed no dosimetric impact of either of the techniques.

\section{Discussion}

The implementation of modern radiotherapy (3D conformal and IMRT) has led to a reduction of the mean total dose to organs at risk and particularly to the optic nerves when compared to conventional radiotherapy [12]. Radiation optic neuropathy is highly dependant on the radiation dose [13-15]. Consequently, the high incidence of radiotherapy-induced blindness, as much as $37 \%$, with conventional radiotherapy could be reduced by the use of $3 \mathrm{D}$ radiotherapy [16-18]. However in a single institution study with 40 patients [19], conformal radiotherapy for paranasal sinus carcinoma seems to be safer with only a 5 $\%$ incidence of cataract and only $2.5 \%$ unilateral blindness.

The benefits, in dose reduction to the optic pathways, of IMRT over 3D conformal radiotherapy have already been investigated [20], with an average maximum dose of 56.4 Gy for IMRT and of 64.2 Gy for 3D conformal radiotherapy. However, a complex treatment planning with 4 -field 3D conformal radiotherapy and forward treatment planning will yield similar results to CF IMRT [21]. We have to point out though, that since high doses were delivered to both optic nerves, this diminishes the case for IMRT in this study.

One other advantage of IMRT is the mode of administration of radiotherapy: as integrated concomitant boosts with a daily dose fraction to OAR of less than 2 Gy which can prevent late complications [14].

In a publication by the M. D. Anderson Cancer Center, the maximum point dose for the controlateral optic nerve was significantly reduced when using 5 non coplanar field IMRT rather than the 9 coplanar field method [22]. On 


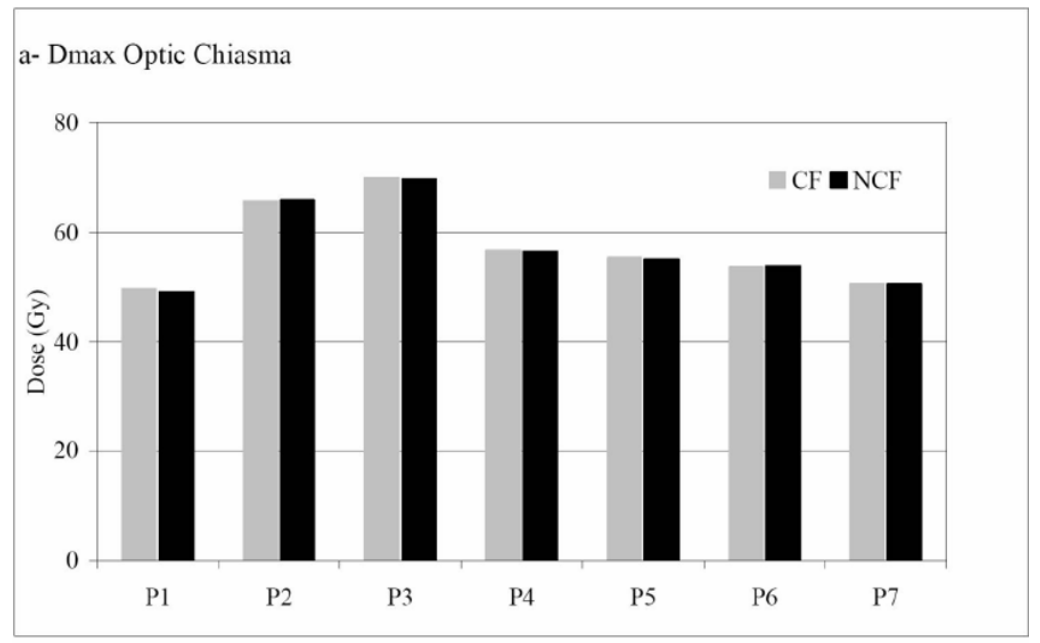

b- Dmax Ipsilateral Optic Nerve
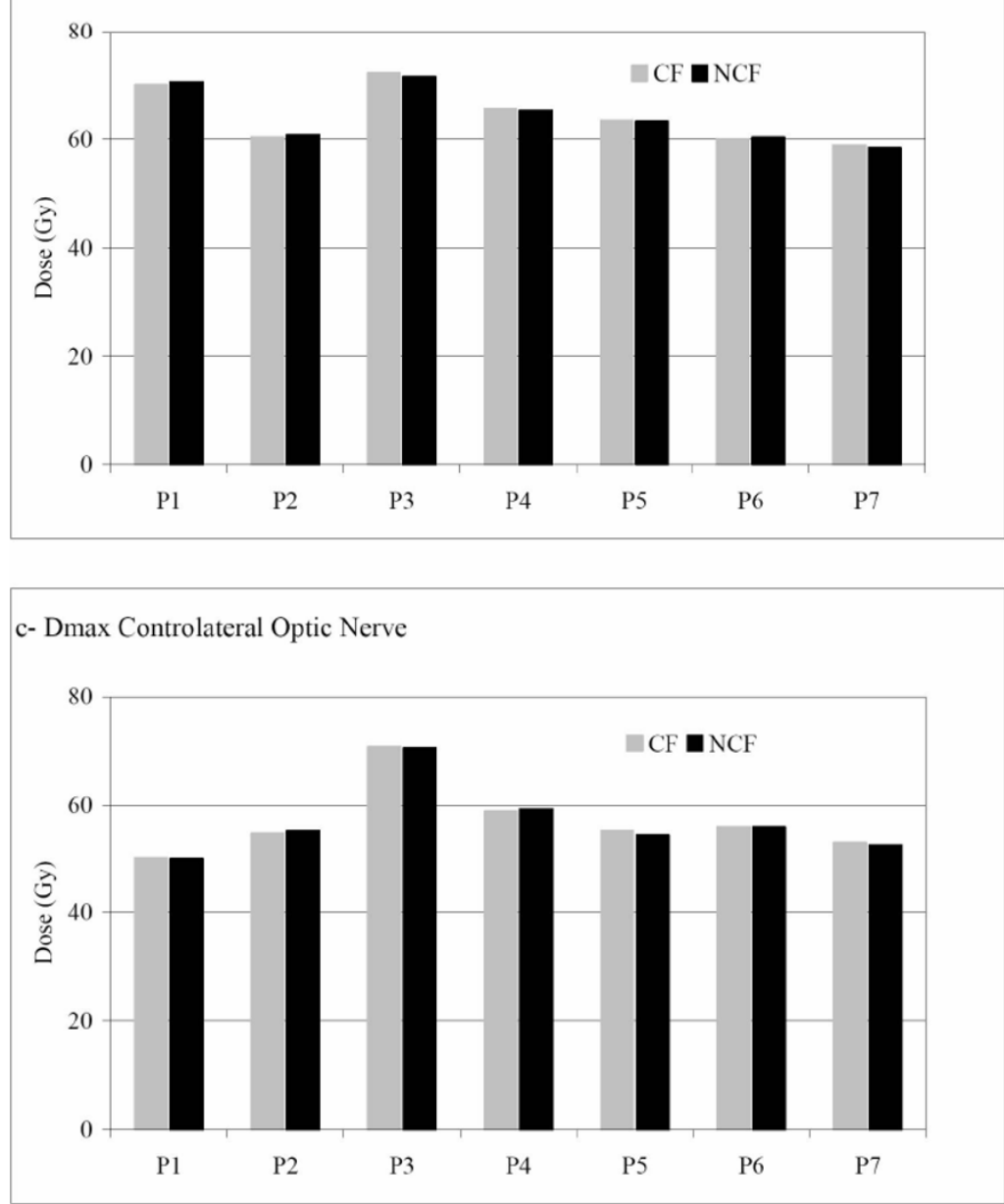

Figure I

Maximum doses in optic pathways, respectively optic chiasma (a), ipsilateral optic nerve (b) and controlateral optic nerve (c). For each patient (Px), the maximum dose for coplanar field CF (grey) and for non coplanar field NCF (black) is represented. 

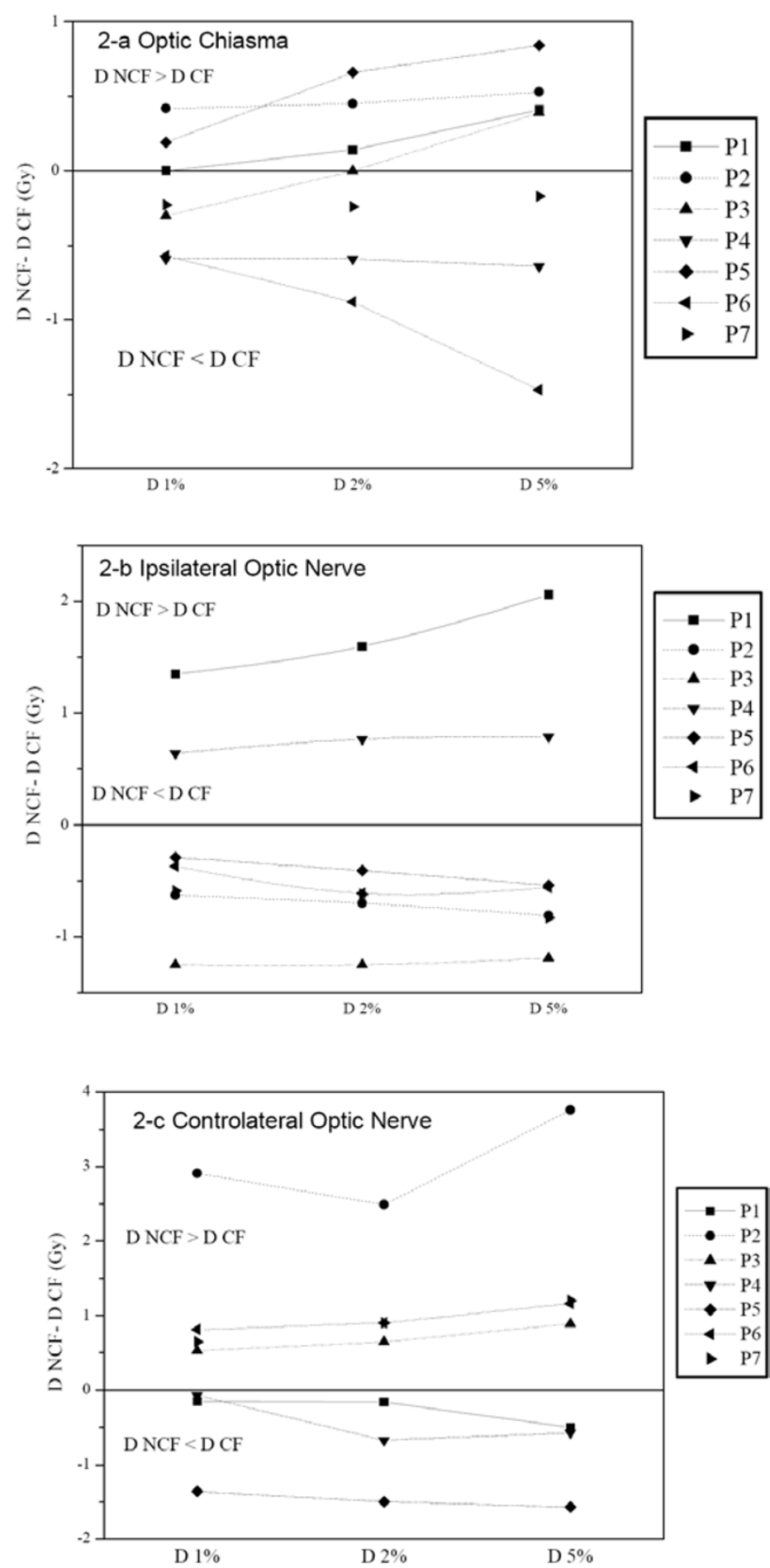

Figure 2

Dose distribution in optic pathways for each patient (Px); optic chiasma (a), ipsilateral optic nerve (b) and controlateral optic nerve (c) respectively. The dose difference in Gy between non coplanar field NCF and coplanar field CF (D NCF - D CF) is represented in terms of DI\%, D2\%, and D5\% corresponding to the doses in I, 2 and $5 \%$ of the volumes respectively. This means that when the difference is negative, the dose to optic pathways is higher when using coplanar field technique. 


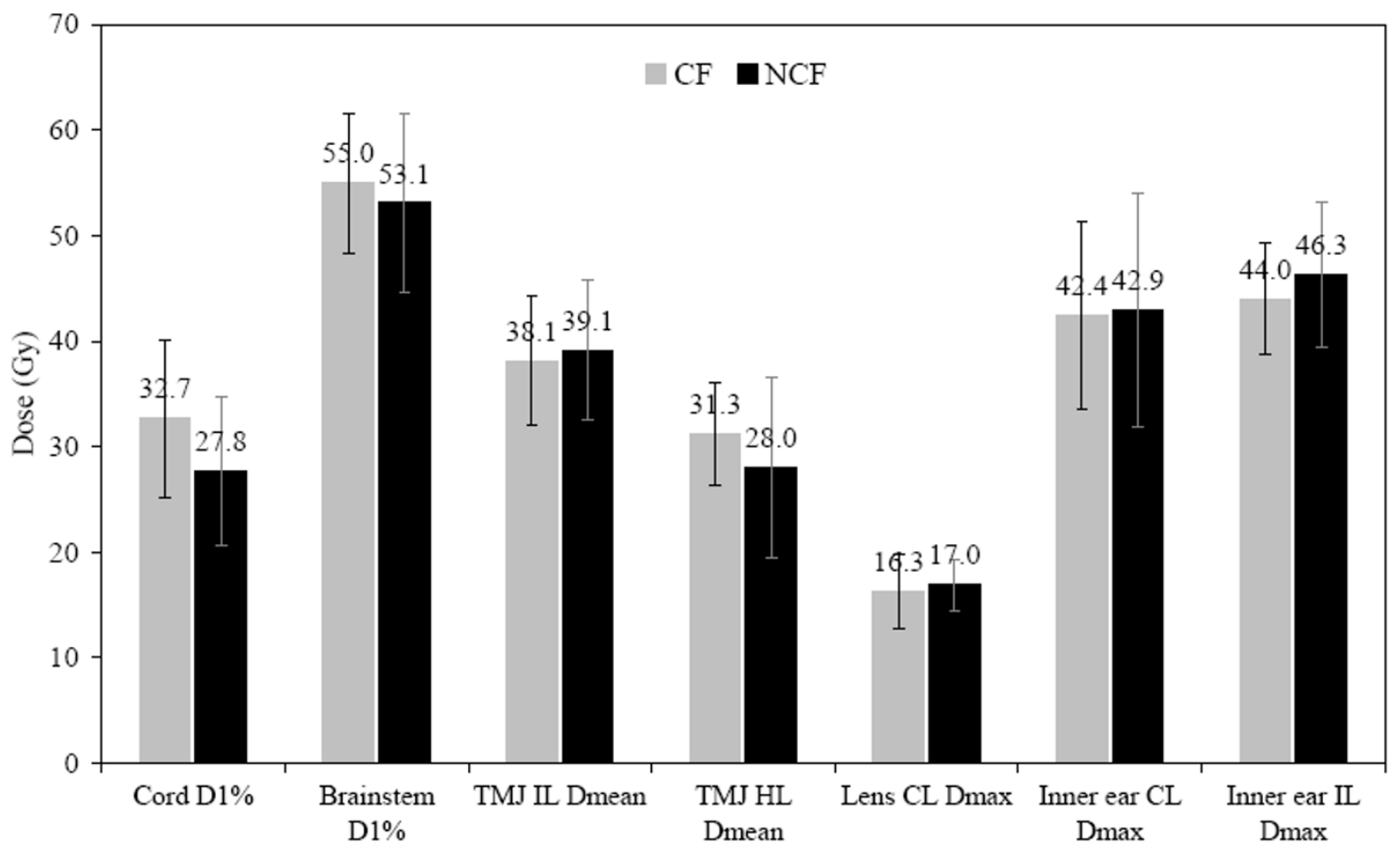

Figure 3

Comparison of dose distribution in organs at risk for the coplanar field technique CF (grey) and non coplanar field technique NCF (black). The most characteristic tolerance dose for each organ at risk is represented. DI\%, Dmean and Dmax are the I\% of organ volume doses, mean dose and maximum dose respectively. Mean dose values and standard deviation are shown.

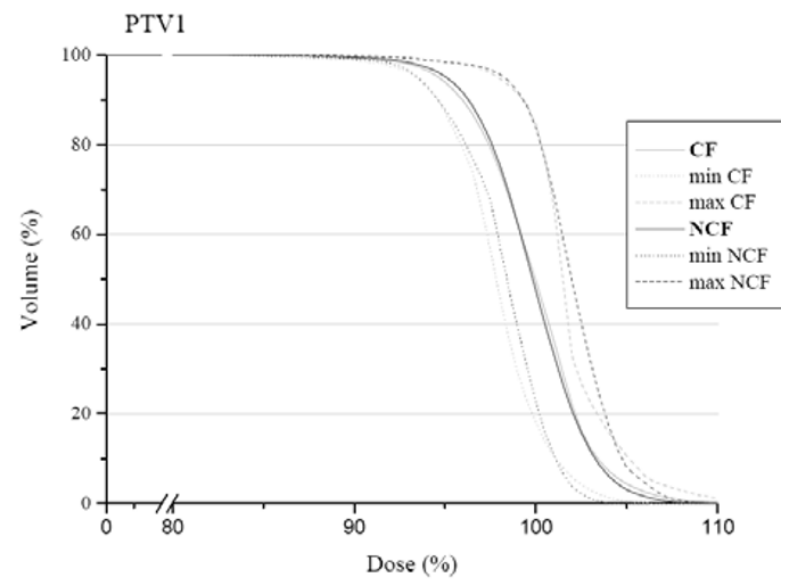

\section{Figure 4}

Mean dose volume histogram in the planning target volume for the coplanar field CF (grey) and non coplanar field technique NCF (black). Minimum and maximum doses of the study group are represented by dotted lines.
Homogeneity Index (HI)

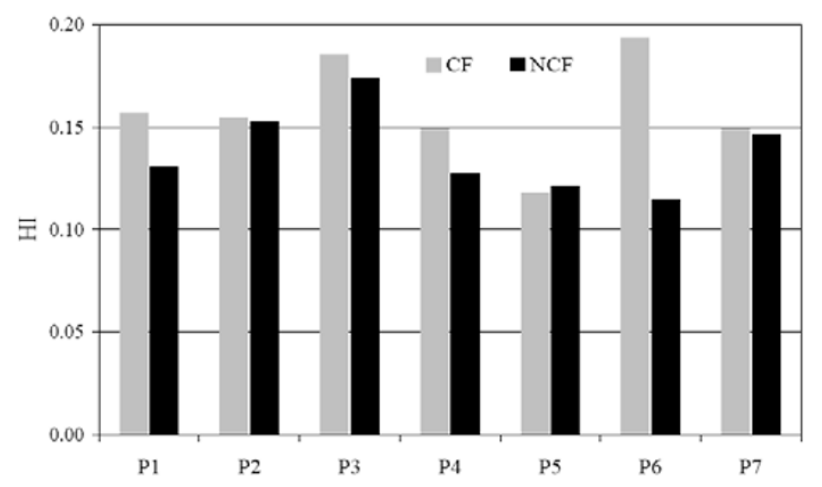

Figure 5

Homogeneity index (HI) for each patient in the coplanar field $\mathrm{CF}$ (grey) and non coplanar field NCF (black) technique. $\mathrm{HI}$ is defined as the difference between DI and D99\% divided by the prescribed dose. A perfect homogeneity would be reached with a zero index. 
Conformity Index (CI)

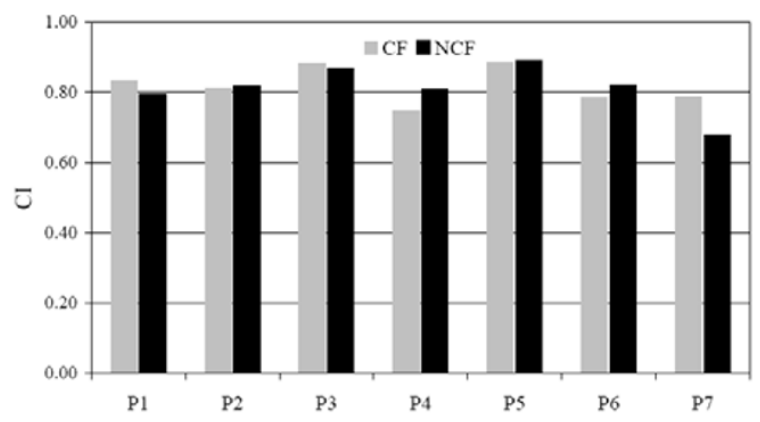

\section{Figure 6}

Conformal index $\mathrm{Cl}$ for each patient in the coplanar field $\mathrm{CF}$ (grey) and non coplanar field NCF (black) technique. $\mathrm{Cl}$ is defined as follows: $\mathrm{Cl}=\left(\mathrm{TV} / \mathrm{V}_{\mathrm{PTV}}\right) \times\left(\mathrm{TV} / \mathrm{V}_{95 \%}\right) \mathrm{TV}$ : Treated Volume is the volume of PTVI receiving the prescribed dose (95\%); $\mathrm{V}_{\mathrm{PTV}}$ is the volume of PTV; $\mathrm{V}_{95 \%}$ is the volume enclosed in the isodose $95 \%$

the other hand, there was no significant difference for the ipsilateral nerve. In our experience, we did not observe better D1, D2 and D5\% to the optic pathways with the non coplanar fields when using the same maximum dose for both techniques.

The significance of maximum point dose to serial organs such as chiasma or optic nerves in fractionated radiotherapy is not well known, and many teams use D1 or D2 as the maximum tolerated dose for the validation of dosim-

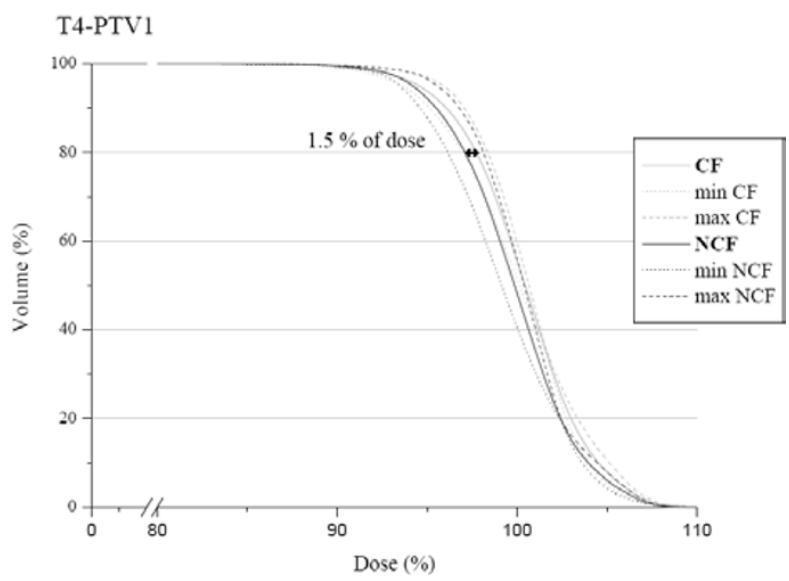

\section{Figure 7}

PTV mean dose volume histogram in the T4 subgroup for the coplanar field CF (grey) and non coplanar field technique NCF (black). Minimum and maximum doses of the study group are represented by dotted lines. etry. A Belgian study by Claus et al. introduced a planning organ at risk volume (PRV) made of a $2 \mathrm{~mm}$ isotropic expansion around the optic pathway with the following constraint: less than 5\% of the PRV should receive more than 60 Gy [23]. A median follow up of 31 months [24] for the 39 patients did not show any radiotherapyinduced blindness. Moreover, a recent publication [25] with 36 patients treated by IMRT for paranasal carcinoma did not report decreased vision.

For small organ volumes such as the lens, we used a PRV with $2 \mathrm{~mm}$ isotropic margin. During optimization, constraints were modified in order to have the lowest dose possible. Maximum dose to the lens was very similar for both techniques, around 16 Gy. Probability of cataract remained high at $27 \%$ at 5 years and $57 \%$ at 8 years for a dose of 15 Gy in 15 fractions, as described by Henk et al. [26].

This excess dose to the lens does not depend on the radiotherapy technique, and even with conformal 3D treatment $[12,21]$, the ipsilateral and controlateral lenses are irradiated more than the tolerated dose given by TD 5/5 published by Emami [27]. Proton therapy could be used to reduce the dose to the controlateral lens but $40 \%$ of the controlateral lens will still be overdosed [12], without any impact on the dose delivered to the ipsilateral lens.

It would be tempting to compare the two treatment modalities for the entire ocular globe. We know that coplanar IMRT and 3D conformal radiotherapy reduce the mean total dose in a similar way when compared to conventional irradiation [12], with a slight benefit with a 5beam conformal radiotherapy over coplanar IMRT [28]. A significant dosimetric advantage represented by the mean dose is noted for non coplanar over coplanar field IMRT when considering the two ocular globes [22]. Nevertheless, the pertinence of these comparisons for clinical practice is doubtful because of the difference in radiosensibility for the diverse components of the eye [29].

The DVH and the conformity index for the PTV1 are similar between the two techniques. No available data demonstrating the superiority of the non coplanar over coplanar fields on the target volume has been published. This lack of difference can be explained by the ease with which the inverse planning system can follow the constraints prescribed for sliding window IMRT. No superiority of conformal 3D treatment over conventional treatment planning was demonstrated in maxillary sinus tumors [20]. In ethmoid carcinoma, conformal radiotherapy with forward planning is better than conventional planning $[21,28]$. 
In the publication of Adams et al., the conformity and homogeneity indices are better with IMRT than with conformal treatment [20]. The same observations were made when comparing multifield dynamic IMRT and step-andshoot IMRT to 3D conformal treatment [30]. Interestingly, no dosimetric benefits for the target volume were noted with IMRT over conformal treatment planning in the maxillary sinus [12], probably due to the distance from the optic pathway and the lack of concave organs.

The MD Anderson Cancer Center experience did not directly compare coplanar and non coplanar field IMRT; however, they used a parallelized multi-resolution beam angle optimization (PMBAO) which included non coplanar fields and obtained thus better dose homogeneity without a real impact on the conformity index [22]. Their results could be explained by the use of only two non coplanar beams in a 5-beam configuration. The impact noted on the homogeneity in the target volume is in agreement with our results and seems to be due to non coplanar fields rather than the PMBAO.

\section{Conclusion}

IMRT using the non coplanar field technique in ethmoid carcinoma is an effective approach for treating this tumor. A slight impact was shown on the PTV coverage for the non coplanar set up compared with the coplanar technique. Using our beam configuration, T4 tumors with skull base involvement were better treated with coplanar fields. In all cases, inverse planning allows for a dosimetric sparing of the optic pathways with good target volume coverage whatever the set up employed. The clinical impact on local control and on late effects is still not known with IMRT and a retrospective analysis of this cohort of patients is required.

\section{Abbreviations}

IMRT- Intensity modulated radiotherapy;

NCF- Non coplanar field;

CF- Coplanar field;

GTV- Gross tumor volume;

CTV- Clinical target volume;

PTV- Planning target volume;

Dmax- Maximum delivered dose;

DVH- Dose-volume histogram;

HI- Homogeneity index;
OAR- Organ at risk;

PMBAO- Parallelized multi-resolution beam angle optimization.

\section{Competing interests}

The author(s) declare that they have no competing interests.

\section{Authors' contributions}

AS, KI, and PF conceived the study, collected data, and drafted the manuscript.

AA, LS, and NA collected data.

JBD and DA participated in coordination and helped to draft the manuscript.

$\mathrm{CL}, \mathrm{RG}$, and MM participated in the design of the study and assisted in data collection.

DA provided mentorship and edited the manuscript.

All authors have read and approved the final manuscript.

\section{Acknowledgements}

The authors would like to thank B. Hawkins and F. Godson for the excellent assistance in the preparation of this manuscript.

\section{References}

I. Dulguerov P, Jacobsen MS, Allal AS, Lehmann W, Calcaterra T: Nasal and paranasal sinus carcinoma: are we making progress? A series of 220 patients and a systematic review. Cancer 200I, 92:3012-3029.

2. Dulguerov P, Allal AS: Nasal and paranasal sinus carcinoma: how can we continue to make progress? Current opinion in otolaryngology \& head and neck surgery 2006, I 4:67-72.

3. Jansen EP, Keus RB, Hilgers FJ, Haas RL, Tan IB, Bartelink H: Does the combination of radiotherapy and debulking surgery favor survival in paranasal sinus carcinoma? Int J Radiat OncolBiol Phys 2000, 48:27-35.

4. Amendola BE, Eisert D, Hazra TA, King ER: Carcinoma of the maxillary antrum: surgery of radiation therapy? Int J Radiat Oncol Biol Phys 198I, 7:743-746.

5. Hordijk GJ, Brons EN: Carcinomas of the maxillary sinus: a retrospective study. Clinical otolaryngology and allied sciences 1985, 10:285-288.

6. Jiang GL, Ang KK, Peters LJ, Wendt CD, Oswald MJ, Goepfert H: Maxillary sinus carcinomas: natural history and results of postoperative radiotherapy. Radiother Oncol |99|, 21:193-200.

7. Jiang GL, Morrison WH, Garden AS, Geara F, Callender D, Goepfert $H$, Ang KK: Ethmoid sinus carcinomas: natural history and treatment results. Radiother Oncol 1998, 49:2I-27.

8. Paulino AC, Fisher SG, Marks JE: Is prophylactic neck irradiation indicated in patients with squamous cell carcinoma of the maxillary sinus? Int J Radiat Oncol Biol Phys 1997, 39(2):283-289.

9. Tsujii H, Kamada T, Arimoto T, Mizoe J, Shirato H, Matsuoka Y, Irie $\mathrm{G}$ : The role of radiotherapy in the management of maxillary sinus carcinoma. Cancer 1986, 57:226I-2266.

10. Tsien C, Eisbruch A, McShan D, Kessler M, Marsh R, Fraass B: Intensity-modulated radiation therapy (IMRT) for locally advanced paranasal sinus tumors: incorporating clinical decisions in the optimization process. Int J Radiat Oncol Biol Phys 2003, 55:776-784. 
II. Feuvret L, Noel G, Mazeron J], Bey P: Conformity index: a review. Int J Radiat Oncol Biol Phys 2006, 64:333-342.

12. Mock U, Georg D, Bogner J, Auberger T, Potter R: Treatment planning comparison of conventional, 3D conformal, and intensity-modulated photon (IMRT) and proton therapy for paranasal sinus carcinoma. Int J Radiat Oncol Biol Phys 2004, 58:147-I54.

13. Jiang GL, Tucker SL, Guttenberger R, Peters LJ, Morrison WH, Garden AS, Ha CS, Ang KK: Radiation-induced injury to the visual pathway. Radiother Oncol 1994, 30:17-25.

14. Parsons JT, Bova FJ, Fitzgerald CR, Mendenhall WM, Million RR: Radiation optic neuropathy after megavoltage external-beam irradiation: analysis of time-dose factors. Int J Radiat Oncol Biol Phys 1994, 30:755-763.

15. Takeda A, Shigematsu N, Suzuki S, Fujii M, Kawata T, Kawaguchi O, Uno $\mathrm{T}$, Takano $\mathrm{H}$, Kubo $\mathrm{A}$, Ito $\mathrm{H}$ : Late retinal complications of radiation therapy for nasal and paranasal malignancies: relationship between irradiated-dose area and severity. Int J Radiat Oncol Biol Phys 1999, 44:599-605.

16. Ellingwood KE, Million RR: Cancer of the nasal cavity and ethmoid/sphenoid sinuses. Cancer 1979, 43:1517-1526.

17. Katz TS, Mendenhall WM, Morris CG, Amdur RJ, Hinerman RW, Villaret DB: Malignant tumors of the nasal cavity and paranasal sinuses. Head Neck 2002, 24:82I-829.

18. Shukovsky LJ, Fletcher GH: Retinal and optic nerve complications in a high dose irradiation technique of ethmoid sinus and nasal cavity. Radiology 1972, 104:629-634.

19. Pommier P, Ginestet C, Sunyach M, Zrounba P, Poupart M, Ceruse P, Ciupea C, Carrie C, Montbarbon X: Conformal radiotherapy for paranasal sinus and nasal cavity tumors: three-dimensional treatment planning and preliminary results in $\mathbf{4 0}$ patients. Int J Radiat Oncol Biol Phys 2000, 48:485-493.

20. Adams EJ, Nutting CM, Convery DJ, Cosgrove VP, Henk JM, Dearnaley DP, Webb S: Potential role of intensity-modulated radiotherapy in the treatment of tumors of the maxillary sinus. Int J Radiat Oncol Biol Phys 200I, 5 I:579-588.

21. Pacholke HD, Amdur RJ, Louis DA, Yang H, Mendenhall WM: The role of intensity modulated radiation therapy for favorable stage tumor of the nasal cavity or ethmoid sinus. Am J Clin Oncol 2005, 28:474-478.

22. Wang X, Zhang X, Dong L, Liu H, Gillin M, Ahamad A, Ang K, Mohan $R$ : Effectiveness of noncoplanar IMRT planning using a parallelized multiresolution beam angle optimization method for paranasal sinus carcinoma. Int J Radiat Oncol Biol Phys 2005, 63:594-601.

23. Claus F, De Gersem W, De Wagter C, Van Severen R, Vanhoutte I, Duthoy W, Remouchamps V, Van Duyse B, Vakaet L, Lemmerling M, Vermeersch $\mathrm{H}$, De Neve W: An implementation strategy for IMRT of ethmoid sinus cancer with bilateral sparing of the optic pathways. Int J Radiat Oncol Biol Phys 200I, 5 I:3 I8-33I.

24. Duthoy W, Boterberg T, Claus F, Ost P, Vakaet L, Bral S, Duprez F, Van Landuyt M, Vermeersch H, De Neve W: Postoperative intensity-modulated radiotherapy in sinonasal carcinoma: clinical results in 39 patients. Cancer 2005, 104:7I-82.

25. Daly ME, Chen AM, Bucci MK, El-Sayed I, Xia P, Kaplan MJ, Eisele DW: Intensity-modulated radiation therapy for malignancies of the nasal cavity and paranasal sinuses. Int J Radiat Oncol Biol Phys 2007, 67:151-157.

26. Henk JM, Whitelocke RA, Warrington AP, Bessell EM: Radiation dose to the lens and cataract formation. Int J Radiat Oncol Biol Phys 1993, 25:815-820.

27. Emami B, Lyman J, Brown A, Coia L, Goitein M, Munzenrider JE, Shank $B$, Solin LJ, Wesson M: Tolerance of normal tissue to therapeutic irradiation. Int J Radiat Oncol Biol Phys 199|, 21:109-122.

28. Huang D, Xia P, Akazawa P, Akazawa C, Quivey JM, Verhey LJ, Kaplan $M$, Lee N: Comparison of treatment plans using intensitymodulated radiotherapy and three-dimensional conformal radiotherapy for paranasal sinus carcinoma. Int J Radiat Oncol Biol Phys 2003, 56:158-168.

29. Nakissa N, Rubin P, Strohl R, Keys H: Ocular and orbital complications following radiation therapy of paranasal sinus malignancies and review of literature. Cancer 1983, 51 :980-986.

30. O'Daniel JC, Dong L, Kuban DA, Liu H, Schechter N, Tucker SL, Rosen I: The delivery of IMRT with a single physical modulator for multiple fields: a feasibility study for paranasal sinus cancer. Int J Radiat Oncol Biol Phys 2004, 58:876-887.
Publish with Biomed Central and every scientist can read your work free of charge

"BioMed Central will be the most significant development for disseminating the results of biomedical research in our lifetime. "

Sir Paul Nurse, Cancer Research UK

Your research papers will be:

- available free of charge to the entire biomedical community

- peer reviewed and published immediately upon acceptance

- cited in PubMed and archived on PubMed Central

- yours - you keep the copyright

Submit your manuscript here:

http://www.biomedcentral.com/info/publishing_adv.asp
BioMedcentral 